\section{ORIGINAL RESEARCH}

\author{
A.R. Sepahdari \\ R. Kapur \\ V.K. Aakalu \\ J.P. Villablanca \\ M.F. Mafee
}

\title{
Diffusion-Weighted Imaging of Malignant Ocular Masses: Initial Results and Directions for Further Study
}

BACKGROUND AND PURPOSE: Ocular masses represent a spectrum of malignant tumors and benign lesions that are sometimes difficult to detect and differentiate by conventional imaging techniques. The aim of this study was to characterize a group of malignant ocular masses with DWI, with the goals of establishing reference data and identifying potential clinical applications for improved noninvasive characterization.

MATERIALS AND METHODS: With institutional review board approval, 26 malignant ocular masses in 22 patients were retrospectively analyzed. Five masses were excluded from further analysis due to nonvisualization. Fifteen retinoblastomas, 5 melanomas, and 1 highly undifferentiated carcinoma were studied. Region-of-interest analysis was performed, and the ADC of each mass was measured and also compared with a normal-appearing thalamus. Lesion thickness was measured, the amount of susceptibility artifact was qualitatively assessed and graded, and the correlation between these factors and retinoblastoma ADC was determined.

RESULTS: Retinoblastomas had an ADC of $0.93 \pm 0.3 \times 10^{-3} \mathrm{~mm}^{2} / \mathrm{s}$ (mean). Melanoma had an ADC of $1.18 \pm 0.16 \times 10^{-3} \mathrm{~mm}^{2} / \mathrm{s}$. The ADC of retinoblastoma was strongly inversely correlated with lesion thickness, likely representing the effect of partial volume averaging. ADC was not correlated with the amount of subjectively determined susceptibility artifact.

CONCLUSIONS: Malignant ocular tumors were consistently characterized with DWI, though with limitations due to artifact and partial volume averaging. Additional description of DWI of ocular masses and further technical improvements may lead to a clinical role for DWI.

ABBREVIATIONS: PROPELLER = periodically rotated overlapping parallel lines with enhanced reconstruction

0 cular masses represent a spectrum of benign and malignant lesions in adults and children that can be challenging to diagnose and treat. Imaging plays an important role in diagnosis, due to a potentially limited clinical examination and risks associated with biopsy. MR imaging is a powerful tool for imaging the globe, due to the excellent tissue contrast it provides.

Although MR imaging frequently yields excellent characterization of globe lesions, there are limitations to the information that MR imaging can provide. Since the initial descriptions of the MR imaging appearance of various ocular masses, continued technical advances in MR imaging have allowed higher resolution imaging and enhanced characterization of lesions. ${ }^{1-5}$ Despite high spatial resolution, MR imaging by using routine sequences is sometimes unable to distinguish different ocular masses and may be limited in its ability to detect ocular masses in the presence of hemorrhagic/exudative reti-

Received March 15, 2011; accepted after revision May 19.

From the Department of Radiological Sciences (A.R.S., J.P.V.), David Geffen School of Medicine, University of California, Los Angeles, Los Angeles, California; Department of Ophthalmology (R.K.), Medical College of Wisconsin, Milwaukee, Wisconsin; Department of Ophthalmology and Visual Sciences (V.K.A.), University of Illinois at Chicago, Chicago, Illinois; and Department of Radiology (M.F.M.), University of California, San Diego, San Diego, California.

Please address correspondence to Ali R. Sepahdari, MD, Department of Radiological Sciences, University of California, Los Angeles, David Geffen School of Medicine, 757 Westwood Plaza, Suite 1621D, Los Angeles, CA 90095; e-mail: ali.sepahdari@gmail.com 三Indicates article with supplemental on-line table. http://dx.doi.org/10.3174/ajnr.A2747 nal detachments, due to suboptimal tissue contrast. Thus, it would be helpful to evaluate additional MR imaging techniques capable of providing different forms of tissue contrast that may aid in the detection and further characterization of ocular masses.

DWI, an MR imaging technique initially used for detection of acute infarction, has been increasingly used to characterize soft-tissue masses in the head and neck, particularly because technical advances such as parallel imaging have helped overcome the challenges of magnetic susceptibility artifacts related to the commonly used EPI technique and have facilitated implementation of rapid scanning algorithms. Several case series and case reports have used DWI to characterize orbital masses, inflammatory lesions, and infection; and additional scattered case reports are available describing the use of DWI to characterize ocular lesions such as endophthalmitis. ${ }^{6-10}$ Isolated examples of DWI of retinoblastoma have also been reported (Castillo M, "More on DWI of Head and Neck Lesions" January 1, 2009; ajnrblog.org). ${ }^{11}$ To our knowledge, there has not yet been a systematic analysis of ocular masses by using DWI.

The purpose of this retrospective study was to characterize a group of malignant ocular masses with DWI by using EPI and parallel imaging, with the goal of establishing reference data that may serve as a baseline for further investigation of DWI as a tool for diagnosis, initial staging, and assessment of treatment response. 


\section{Materials and Methods}

\section{Subjects}

Institutional review board approval and a waiver of informed consent were obtained for this retrospective study. A database of orbital and ocular lesions, created by consecutive review of imaging studies from 2000 to 2009, was queried. Patients with enhancing intraocular masses who had not yet received treatment were identified. Twenty-six ocular masses were identified in 22 patients, each of whom underwent MR imaging with EPI DWI by using parallel acquisition. Diagnoses were verified through a review of the electronic medical record, including surgical pathology reports and clinical notes, by an ophthalmologist (V.K.A.), and the images were reviewed by a senior neuroradiologist experienced in head and neck imaging (M.F.M.). The patient cohort was composed of 12 females and 10 males, with an average age of 26 years (range, 4 months to 84 years). The group was bimodal, comprising 12 infants ( 4 months to 2 years of age) with 16 retinoblastomas, one 16-year-old boy with a highly undifferentiated carcinoma (presumptively a dedifferentiated medulloepithelioma), and 9 adults (41-84 years of age) with 7 ocular melanomas, 1 ocular metastasis from colon cancer, and 1 unknown lesion in a patient who was lost to follow-up.

\section{Imaging Technique}

Imaging was performed at $1.5 \mathrm{~T}$ in 12 patients (Signa Excite; GE Healthcare, Milwaukee, Wisconsin) and at $3 \mathrm{~T}$ in 10 patients (Signa Excite), by using an 8-channel head coil. DWI was performed in the transverse plane by using a single-shot spin-echo EPI sequence with array spatial sensitivity encoding technique (ASSET) parallel acquisition. b-values of 0 and $1000 \mathrm{~s} / \mathrm{mm}^{2}$ were used. One signal was acquired at $1.5 \mathrm{~T}$, and 2 signals, at 3T. All images were acquired with a $128 \times 128$ matrix. TR, TE, FOV, section thickness, and intersection gap varied between studies. Average TRs/TEs were $8000 / 76 \mathrm{~ms}$ at $1.5 \mathrm{~T}$, and $6660 / 69 \mathrm{~ms}$ at $3 \mathrm{~T}$. FOV ranged from 20 to $28 \mathrm{~cm}$ based on patient size. Section thickness and intersection gap ranged from 4 to $5 \mathrm{~mm}$ and 0 to $1.5 \mathrm{~mm}$, respectively. Automated ADC maps were created in 19 of 22 cases. Each patient also underwent high-resolution conventional orbital imaging, including 3-mm thin-section fast spin-echo T2-weighted imaging, precontrast axial T1-weighted imaging, and multiplanar postcontrast T1-weighted imaging following infusion of $0.1-\mathrm{mmol} / \mathrm{kg}$ dose of gadodiamide intravenous contrast. All infants in this study were imaged with conscious sedation by using a weight-based dose of oral chloral hydrate.

\section{Image Analysis}

The images were transferred from the PACS server to a hard drive and made anonymous for analysis by using the Osirix Digital Imaging and Communications in Medicine viewer for Macintosh (Osirix, Version 3.5.1; the Osirix Foundation, Geneva, Switzerland). ${ }^{12}$ A fellowshiptrained neuroradiologist, with prior experience in head and neck DWI image analysis and 1 year of practice experience (A.R.S.), performed the image analysis. In each case, the high-resolution conventional T2-weighted images were first assessed. The lesion thickness was determined by drawing a line tangent to the surface of the globe. The lesion was then identified on the ADC map through multiplanar cross-reference with conventional images. The quantitative ADC value was determined by drawing a freehand region of interest over the lesion on the ADC map image, excluding the most peripheral voxels and excluding areas of necrosis evident on the ADC map. No attempt was made to exclude areas of calcification. For the lesions without an automated ADC map, ADC was determined by manual calculation from identical freehand regions of interest drawn on the $b=1000 \mathrm{~s} / \mathrm{mm}^{2}$ and $b=0 \mathrm{~s} / \mathrm{mm}^{2}$ images by using a previously described technique. ${ }^{9}$ For each case, the ADC of the normal-appearing ipsilateral thalamus was also measured, and the ratio of lesion to thalamic ADC was calculated. The thalamus was selected as an internal reference because it exhibits less age-related change in ADC and higher interobserver reproducibility in measurement, compared with normal white matter. ${ }^{13,14}$

The degree of magnetic susceptibility artifact was also graded on a qualitative scale. Warping or distortion of the globe by magnetic susceptibility effect was used to qualitatively estimate the degree of artifact present. Mild globe warping without areas of gross signal-intensity loss was graded as "minimal," moderate globe warping or scattered areas of signal-intensity loss were graded as "mild," moderate globe warping and scattered areas of signal-intensity loss were graded as "moderate," and gross warping with complete obscuration of the globes was graded as "severe." Lesions that could be clearly identified on DWI images and on ADC maps (when available) were considered "well characterized."

\section{Statistical Analysis}

Lesions were grouped by diagnosis. The mean and SD of the ADC values for each lesion were determined. For the largest group of lesions (retinoblastoma), quantitative lesion ADC was also plotted against lesion thickness, and regression analysis was performed to determine the relationship between measured ADC and lesion thickness. Spearman rank-order correlation was used to determine whether there was a relationship between the warping artifacts score and measured ADC values. A $P$ value $<.05$ was statistically significant.

\section{Results}

All lesions were well-visualized on conventional images. Five of 26 lesions could not be clearly identified with DWI (2 melanomas, 1 colon cancer metastasis, 1 retinoblastoma, and 1 lesion that was lost to follow-up). These lesions were all between 2 and $3 \mathrm{~mm}$ in thickness. The degree of artifacts was minimal in 2 of these cases, mild in 1 case, and moderate in 2 cases. The remaining 21 lesions ranged from 2 to $15 \mathrm{~mm}$ in thickness. Nine of 10 lesions imaged at 3T and 11 of 15 lesions imaged at $1.5 \mathrm{~T}$ were identified on the ADC maps. ADC maps were available for 20 of these 21 lesions. An ADC map was not created for 1 lesion that was well-visualized on DWI at 3T. One retinoblastoma showed a moderate-sized area of necrosis on DWI and ADC, while the remaining lesions had a homogeneous DWI appearance. Six lesions showed coarse areas of marked T2 hypointensity that presumptively represented calcification. DWI and ADC map signals were homogeneous throughout these lesions.

Of the lesions that were well-visualized, 5 melanomas, averaging $6.6 \mathrm{~mm}$ in thickness, showed an ADC of $1.18 \pm 0.16 \times$ $10^{-3} \mathrm{~mm}^{2} / \mathrm{s}$ (mean). Fifteen retinoblastomas, averaging $8 \mathrm{~mm}$ in thickness, had an ADC of $0.93 \pm 0.3 \times 10^{-3} \mathrm{~mm}^{2} / \mathrm{s}$. One highly undifferentiated carcinoma (presumably dedifferentiated medulloepithelioma) measuring $7 \mathrm{~mm}$ in thickness had an ADC of $1.21 \times 10^{-3} \mathrm{~mm}^{2} / \mathrm{s}$. On-line Table shows detailed results for all lesions, and the Table summarizes the results for lesions that were well-visualized. Figures 1 and 2 show examples of ocular melanoma, Fig 3 shows an example of a retino- 
Table: Summary results for lesions well-characterized by DWI ( $n=21 / 26)$

\begin{tabular}{lccc}
\hline Lesion (No.) & $\begin{array}{c}\text { Average Thickness } \\
\text { (cm) }\end{array}$ & $\begin{array}{c}\text { ADC }\left(10^{-3} \mathrm{~mm}^{2} / \mathrm{s}\right) \\
\text { (mean) }\end{array}$ & $\begin{array}{c}\text { Lesion:Thalamus ADC Ratio } \\
\text { (mean) }\end{array}$ \\
\hline Retinoblastoma (15) & 0.8 & $0.93 \pm 0.30$ & $1.12 \pm 0.35$ \\
Melanoma (5) & 0.7 & $1.18 \pm 0.16$ & $1.65 \pm 0.18$ \\
Highly undifferentiated carcinoma (1) & 0.7 & 1.21 & 1.72 \\
\hline
\end{tabular}
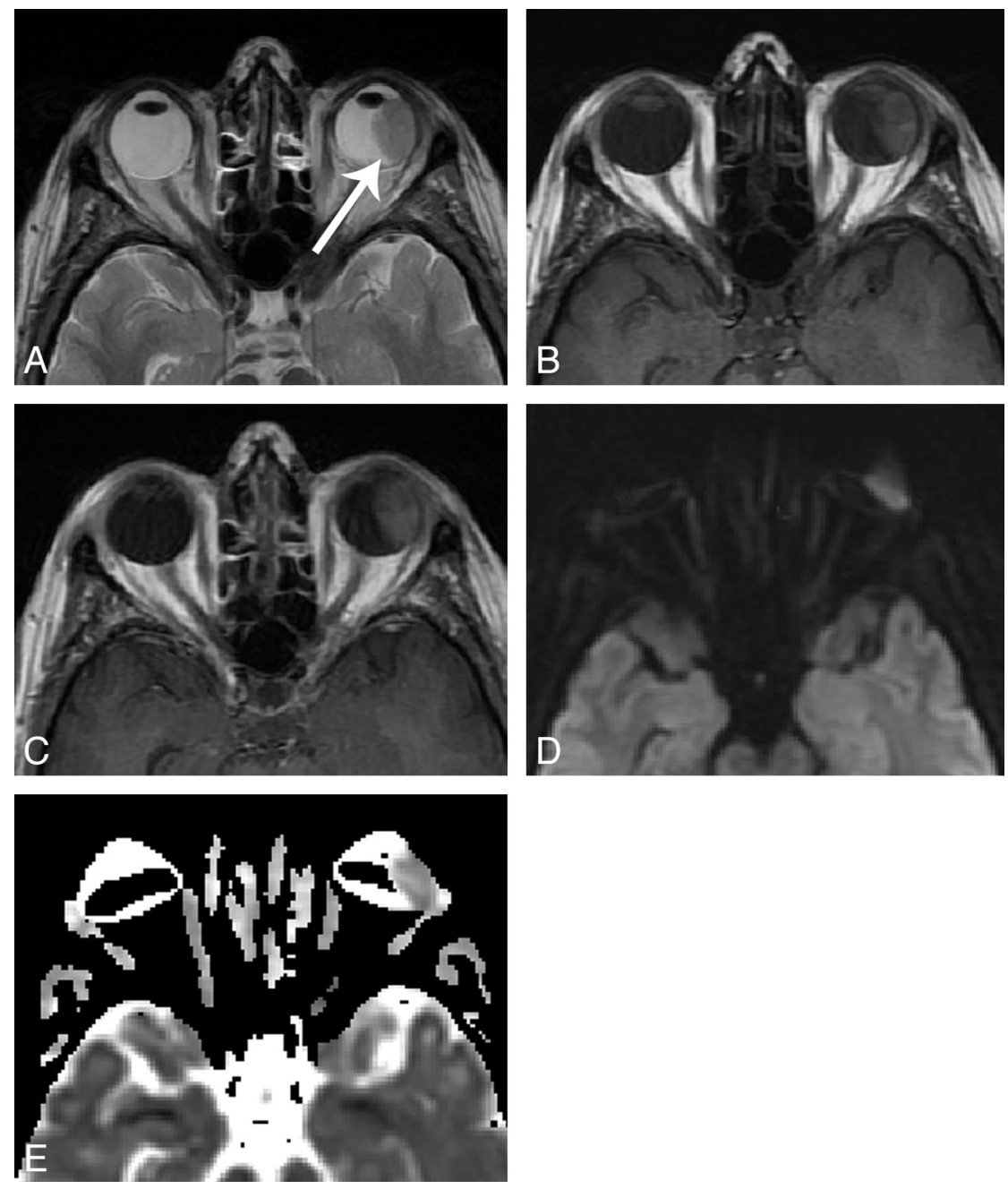

Fig 1. A 48 year-old woman with uveal melanoma, imaged at 3T. $A$, axial T2-weighted image shows a hypointense mass along the temporal aspect of the left globe (arrow). $B$, axial T1-weighted image shows intrinsic T1 hyperintensity. $C$, axial contrast-enhanced T1-weighted image shows moderate, homogeneous contrast enhancement. $D$, axial DWI image shows signal intensity similar to normal gray matter. Slightly lower signal at the anterior margin of the mass may relate to susceptibility artifact as there is some degree of globe warping. $E$, ADC map shows globe warping and areas of signal loss, with a measured ADC of $1.29 \times 10^{-3} \mathrm{~mm}^{2} / \mathrm{s}$. Artifact was graded as "moderate" in this case. (Panels $A$ and $B$ reproduced with permission from Head and Neck Imaging, $5^{\text {th }}$ Ed., Eds: Som PM, Curtin HD. "Pathology of the Eye and Orbit," Cunnane ME, Sepahdari AR, Gardiner M, Mafee MF, p. 632. Copyright Elsevier, 2011.)

blastoma tumor, and Fig 4 shows a highly undifferentiated carcinoma (presumably dedifferentiated medulloepithelioma).

There was no significant relationship between the warping artifacts score and the calculated ADC values. There was a strong inverse relationship $(P<.001)$ between ADC and lesion thickness for retinoblastoma, which best fit a quadratic relationship on regression analysis $\left(r^{2}=0.62\right.$, Fig 5$)$.

\section{Discussion}

Despite varying degrees of magnetic susceptibility artifacts inherent in the EPI technique, $81 \%(21 / 26)$ of the lesions in this group could be characterized with DWI. ADC of retinoblastoma was strongly inversely correlated with lesion size, likely due to partial volume averaging. Notably, many of these lesions were smaller than the limit of the nominal z-axis resolution, ensuring some degree of partial volume averaging that likely resulted in falsely elevated measured ADC for smaller retinoblastoma tumors, as well as a falsely broad distribution of ADC values. The "true" ADC of retinoblastoma is likely lower than that observed in this group, with a smaller range.

Although T1-, T2-, and contrast-enhanced T1-weighted sequences can often distinguish various ocular neoplasms from simulating lesions, certain diagnostic dilemmas remain. MR imaging is frequently used to assess retinoblastoma tumor extent and has room for improvement despite good overall performance. ${ }^{4}$ In the authors' experience, and according to de 

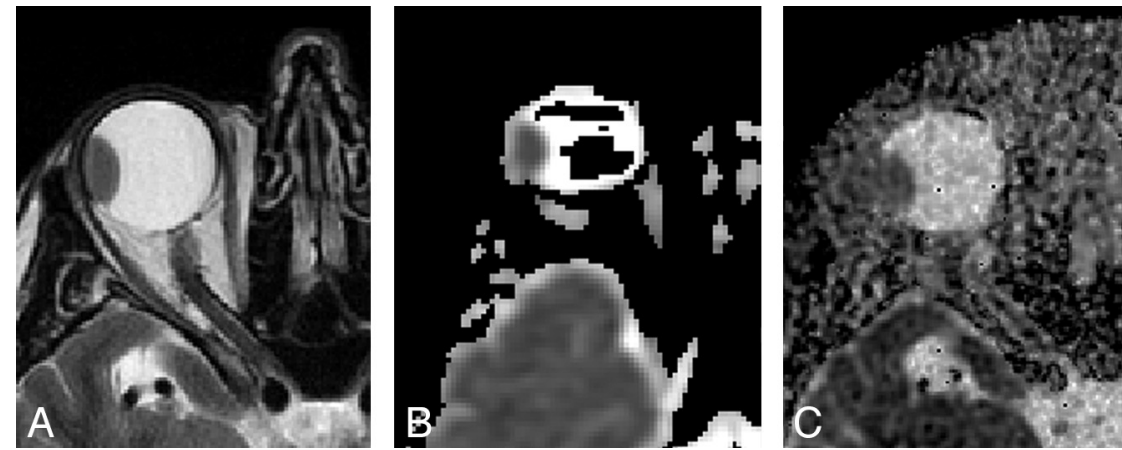

Fig 2. A 63-year-old man with uveal melanoma, imaged at 1.5T. A, Axial T2-weighted image shows a hypointense mass along the temporal aspect of the globe. $B$, Axial ADC map obtained with an EPI technique demonstrates the mass but also shows globe warping and signal-intensity voids in the vitreous, with artifact graded as moderate. ADC was $0.92 \times 10^{-3} \mathrm{~mm}^{2} / \mathrm{s}$. C. Axial ADC map by using the PROPELLER technique shows no evidence of susceptibility artifact. Extensive background noise is present, but there is high contrast between the high-ADC vitreous and low-ADC mass.
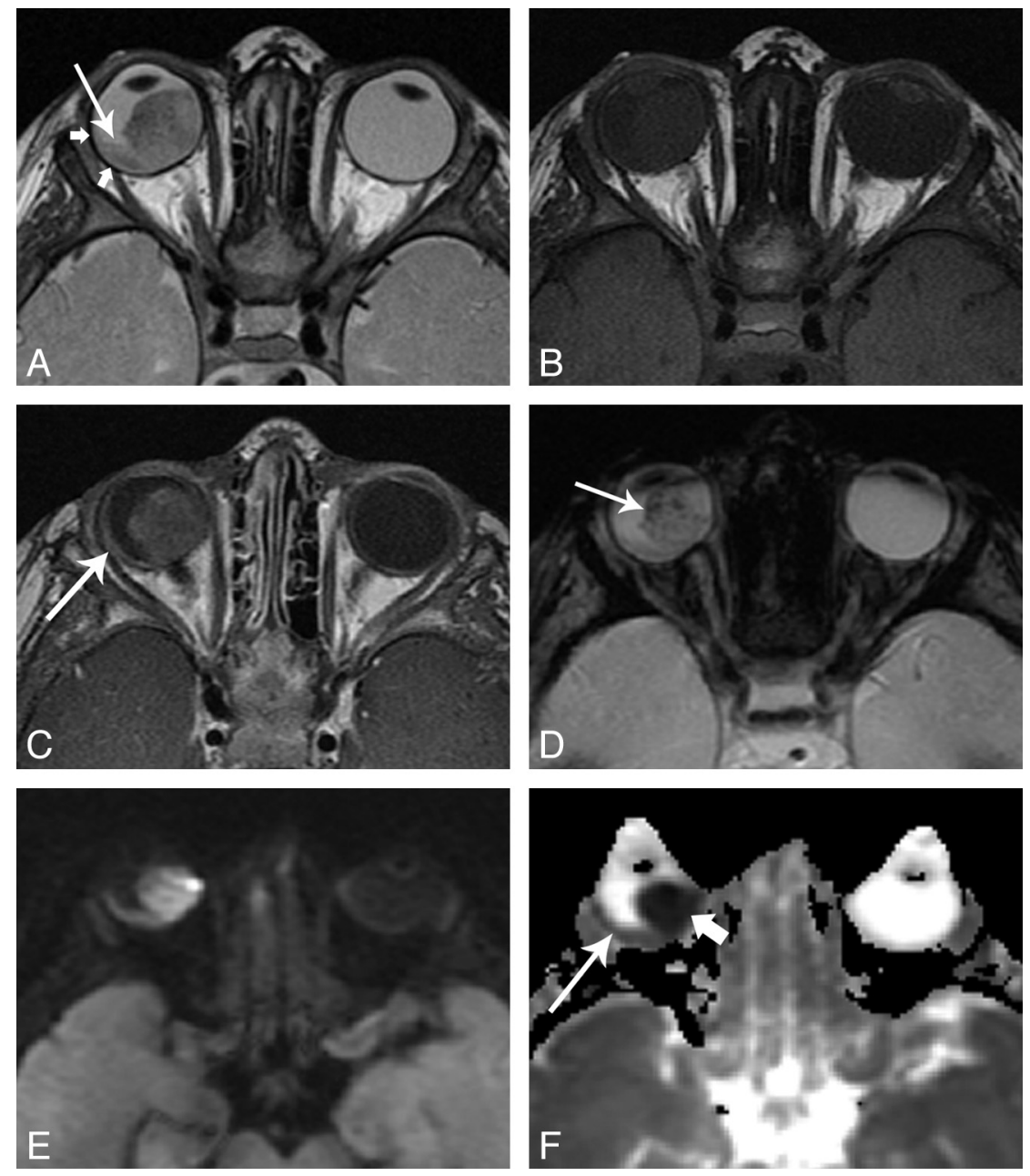

Fig 3. A 15 month-old girl with retinoblastoma, imaged at $3 T$. $A$, axial T2-weighted image shows a large hypointense mass in the right globe. Plaque-like hypointensity along the temporal aspect of the globe represents infiltrating tumor (short arrows). A subtle fluid level at the posterior globe represents associated retinal detachment (/ong arrow). B, axial T1-weighted image shows mild hyperintensity characteristic of retinoblatoma. $C_{1}$ axial contrast-enhanced T1-weighted image shows moderate, slightly heterogeneous enhancement. Note the enhancement of the plaque-like area seen in $A$, without enhancement of the subretinal fluid seen in $A$. $D$, axial T2*-weighted gradient refocused echo-image shows numerous punctate foci of signal loss representing calcifications. $E$, axial DWI image shows homogeneous signal hyperintense to normal gray matter. $F$, ADC map shows markedly lower values than normal brain in the dominant mass (short arrow), with an ADC value slightly lower than normal brain within the thinner portion of the tumor (long arrow). (Panels $A, B$ and $D$ reproduced with permission from Head and Neck Imaging, $5^{\text {th }}$ ed., Eds: Som PM, Curtin HD. "Pathology of the Eye and Orbit," Cunnane ME, Sepahdari AR, Gardiner M, Mafee MF, p. 612. Copyright Elsevier, 2011.).

Potter et al, ${ }^{15}$ uveal melanoma can be indistinguishable from metastasis, and retinoblastoma can be indistinguishable from medulloepithelioma. Despite classically described MR imaging findings that differentiate retinoblastoma from Coat dis- ease, there continue to be occasional cases in which these lesions are indistinguishable despite an exhaustive diagnostic work-up, as reported in the literature and also in the authors' experience. $^{16,17}$ 

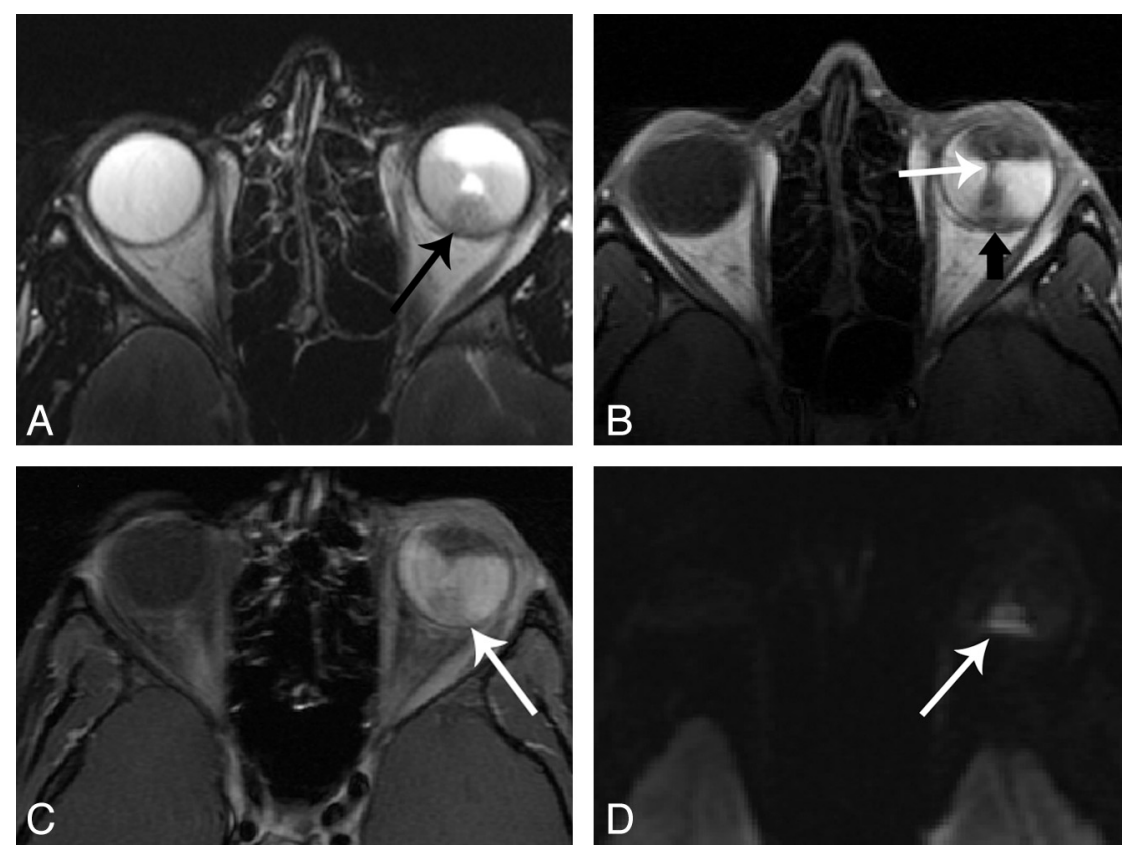

Fig 4. A 16-year-old boy with highly undifferentiated carcinoma (presumptively a dedifferentiated medulloepithelioma), imaged at 3T. A, Axial T2-weighted image shows a hypointense mass at the posteroinferior globe. $B$, Axial T1-weighted image shows an isointense mass (black arrow) in the background of hyperintense exudative retinal detachment. The retinal leaves are elevated and apposed (white arrow). C, Axial contrast-enhanced fat-suppressed T1-weighted image show moderate enhancement of the mass (arrow). D, Axial DWI shows sharp contrast between the cellular mass and the subretinal exudate.

\section{Retinoblastoma}

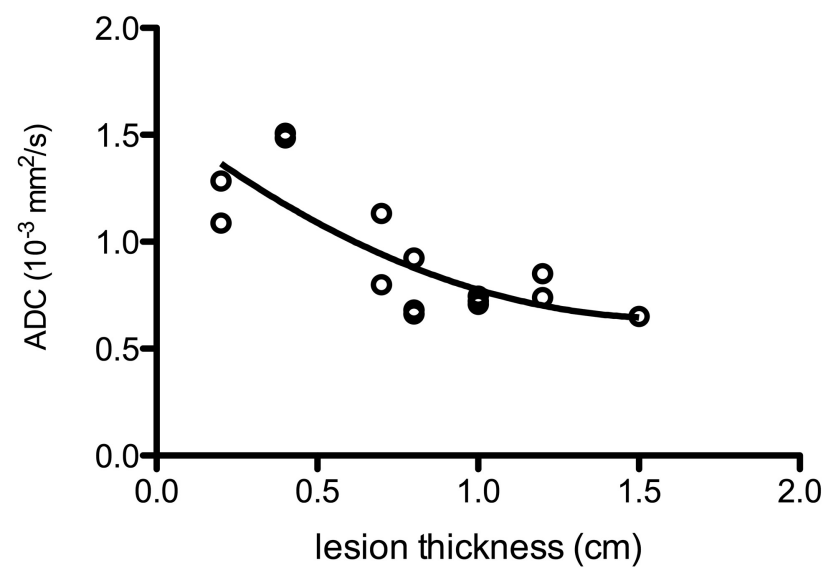

Fig 5. A plot of $A D C$ against lesion thickness for 15 cases of retinoblastoma shows a distribution that best fits a quadratic relationship. Lesions thinner than $0.8-1 \mathrm{~cm}$ were all below the nominal z-axis resolution of our technique and thus were affected by partial volume averaging.

The results of this preliminary investigation provide baseline reference data on the DWI characteristics of the 2 most common ocular malignancies. We were able to confirm that retinoblastoma consistently demonstrates low ADC and that retinoblastoma lesions were not obscured on DWI by calcification. These data may aid further investigation of DWI as a tool for differentiating ocular tumors, differentiating ocular tumors from nontumoral lesions, helping select appropriate therapy, and assessing treatment response in ocular tumors. DWI may also prove useful in detecting small ocular masses that are obscured by subretinal exudates. Figure 4 is an example of a case in which DWI increased the conspicuity of a small tumor within a background of exudative retinal detachment that obscures the lesion on routine sequences.

There are several limitations to this study. First, the lesions that were studied were nearly all melanoma or retinoblastoma, without a large number of simulating lesions for comparison. It is thus impossible to determine whether DWI differentiates lesions with similar clinical and imaging appearance, such as melanoma compared with metastasis or retinoblastoma compared with Coat disease (with enhancing intraocular tissue). Second, most of these lesions were smaller than the nominal $\mathrm{z}$-axis resolution of the DWI technique that was used; this difference ensured some degree of partial volume averaging effect with the potential to impact measured ADC values, as was clearly demonstrated with the group of 15 retinoblastomas. Third, some degree of artifact was present in all cases, with unclear effects on measured ADC. Although susceptibility artifact is typically worst near air-bone interfaces, globe warping is also consistently demonstrated with EPI DWI. The combination of small lesion size and susceptibility artifact resulted in nonvisualization of 19\% (5 of 26) of the lesions imaged on DWI sequences, a higher proportion than has been demonstrated with extraocular orbital lesions $>1 \mathrm{~cm}^{9,10}$ Fourth, a single observer performed all measurements. Although high intraobserver agreement has been previously reported for determination of ADC in orbital masses, the results may be less reproducible for small lesions. ${ }^{9}$ Finally, heterogeneity in the equipment and scanning techniques used may have introduced additional variability in the data. A consistent scanning technique may have resulted in a tighter distribution of data.

Before our findings can be extended to routine clinical practice, an additional description of the DWI features of other ocular lesions is necessary. Technical advances could also help overcome the challenges of susceptibility artifacts 

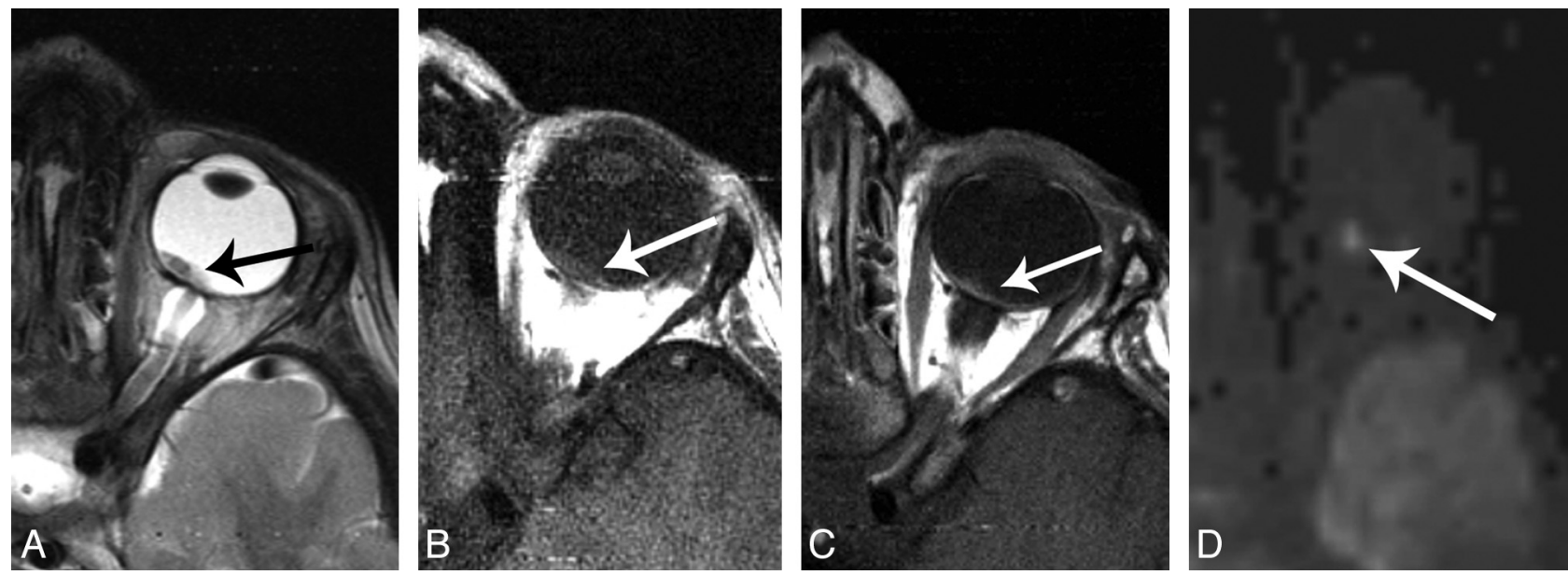

Fig 6. A 3-year-old boy with retinoblastoma, imaged at 3T. $A$, Axial T2-weighted image shows a small hypointense mass at the posterior globe near the optic nerve head. $B$, Axial T1-weighted image shows isointense signal intensity. C, Axial contrast-enhanced T1-weighted image shows uniform enhancement. D, High-resolution axial DWI image obtained with 2-mm isotropic voxels clearly demonstrates DWI signal intensity brighter than normal gray matter in the same section.

and partial volume averaging with small lesions. Potential avenues of exploration include improved EPI and non-EPI DWI techniques. ${ }^{18-21}$ Figure 2 shows an example of a melanoma imaged with EPI DWI and with DWI by using PROPELLER, which uses fast spin-echo acquisition and central $k$-space oversampling, reducing susceptibility artifact and artifact related to gross eye motion. Despite a trade-off in signal-tonoise ratio with PROPELLER DWI, the high-signal-intensity, high ADC vitreous humor provides excellent background for detection of a solid mass. Figure 6 shows an example of a DWI study acquired with 2-mm isotropic voxels by using an EPI DWI sequence with a 12-channel head coil on a 3T system, with excellent visualization of a small residual retinoblastoma lesion that was previously treated with chemotherapy.

\section{Conclusions}

This preliminary study shows that MR imaging by using DWI sequences was able to characterize ocular melanoma and ocular retinoblastoma tumors. Some variability in ADC could be attributed to partial volume averaging. Warping artifacts were also present, with unclear effects on ADC. The population of subjects studied and the DWI technique used do not allow immediate clinical application of these findings. However, these results provide a foundation for further investigation and may be clinically useful in the diagnosis and characterization of ocular lesions when combined with the results of additional prospective and retrospective investigations.

\section{References}

1. Mahajan A, Crum A, Johnson MH, et al. Ocular neoplastic disease. Semin Ultrasound CT MRI 2011;32:28-37

2. Daftari I, Aghaian E, O'Brien JM, et al. 3D MRI-based tumor delineation of ocular melanoma and its comparison with conventional techniques. Med Phys 2005;32:3355-62

3. Apushkin MA, Apushkin MA, Shapiro MJ, et al. Retinoblastoma and simulating lesions: role of imaging. Neuroimaging Clin N Am 2005;15:49-67

4. de Graaf P, Barkhof F, Moll AC, et al. Retinoblastoma: MR imaging parameters in detection of tumor extent. Radiology 2005;235:197-207
5. Chung EM, Specht CS, Schroeder JW. From the archives of the AFIP: pediatric orbit tumors and tumorlike lesions: neuroepithelial lesions of the ocular globe and optic nerve. Radiographics 2007;27:1159-86

6. Sepahdari AR, Aakalu VK, Kapur R, et al. MRI of orbital cellulitis and orbital abscess: the role of diffusion-weighted imaging. AJR Am J Roentgenol 2009; 193:W244-50

7. Kapur R, Sepahdari AR, Mafee MF, et al. MR imaging of orbital inflammatory syndrome, orbital cellulitis, and orbital lymphoid lesions: the role of diffusion-weighted imaging. AJNR Am J Neuroradiol 2009;30:64-70

8. Rumboldt Z, Moses C, Wieczerzynski U, et al.. Diffusion-weighted imaging, apparent diffusion coefficients, and fluid-attenuated inversion recovery MR imaging in endophthalmitis. AJNR Am J Neuroradiol 2005;26:1869-72

9. Sepahdari AR, Aakalu VK, Setabutr P, et al. Indeterminate orbital masses: restricted diffusion at MR imaging with echo-planar diffusion-weighted imaging predicts malignancy. Radiology 2010;256:554-64

10. Politi LS, Forghani R, Godi C, et al. Ocular adnexal lymphoma: diffusionweighted MR imaging for differential diagnosis and therapeutic monitoring. Radiology 2010;256:565-74

11. Mafee MF, Rapoport M, Karimi A, et al. Orbital and ocular imaging using 3 and 1.5-T MR imaging systems. Neuroimaging Clin N Am 2005;15:1-21

12. Rosset A, Spadola L, Ratib O. OsiriX: an open-source software for navigating in multidimensional DICOM images. J Digit Imaging 2004;17:205-16. Epub 2004 Jun 29

13. Morriss MC, Zimmerman RA, Bilaniuk LT, et al. Changes in brain water diffusion during childhood. Neuroradiology 1999;41:929-34

14. Bilgili $Y$, Unal B. Effect of region of interest on interobserver variance in apparent diffusion coefficient measures. AJNR Am J Neuroradiol 2004;25:108-11

15. de Potter P, Flanders AE, Shields JA, et al. The role of fat-suppression technique and gadopentetate dimeglumine in magnetic resonance imaging evaluation of intraocular tumors and simulating lesions. Arch Ophthalmol 1994;112:340-48

16. Forl B, Schmack I, Grossniklaus HE, et al. Coats' disease: important differential diagnosis for retinoblastoma [in German]. Ophthalmologe 2008;105:761-64

17. Dhoot DS, Weissman JL, Landon RE, et al. Optic nerve enhancement in Coats disease with secondary glaucoma. J AAPOS 2009;13:301-02

18. Holdsworth SJ, Skare S, Newbould RD, et al. Robust GRAPPA-accelerated diffusion-weighted readout-segmented (RS)-EPI. Magn Reson Med 2009;62:1629-40

19. Porter DA, Heidemann RM. High resolution diffusion-weighted imaging using readout-segmented echo-planar imaging, parallel imaging and a two-dimensional navigator-based reacquisition. Magn Reson Med 2009;62:468-75

20. De Foer B, Vercruysse J-P, Bernaerts A, et al. Middle ear cholesteatoma: nonecho-planar diffusion-weighted MR imaging versus delayed gadolinium-enhanced T1-weighted MR imaging-value in detection. Radiology 2010;255:866-72

21. Pipe JG, Farthing VG, Forbes KP. Multishot diffusion-weighted FSE using PROPELLER MRI. Magn Reson Med 2002;47:42-52 